\title{
Mixed strategies in PQ-duopolies
}

\author{
D. Cracau $^{a}$, B. Franz ${ }^{b}$ \\ ${ }^{a}$ Faculty of Economics and Management, University of Magdeburg, Universitätsplatz 2, 39106 \\ Magdeburg, Germany \\ ${ }^{\mathrm{b}}$ Mathematical Institute, University of Oxford, 24-29 St Giles', Oxford, OX1 3LB, United Kingdom \\ Email:cracau@ovgu.de
}

\begin{abstract}
The theoretical analysis of complex market competitions is an exceedingly important aspect of modern applied game theory. In general, competition is either modeled to focus on quantities or on prices. The pure strategy equilibria for both games are well known from the literature. A lot of research has also been conducted on broadening assumptions or integrating complex enhancements into these games. Moreover, experimental data have been shown to support the main theoretical results concerning these games. A similar game with prices and quantities as decision variables, however, has not been studied as extensively. So far, no experimental analysis of a standard oligopoly setting with two firms deciding simultaneously on a price and on a quantity has been conducted.

With this paper, we try to fill this gap, as we look at a simple price-quantity (PQ) game and thereby have two major objectives: $(i)$ we establish a numerical solution for the mixed strategy equilibrium in a simplified, discrete version of this PQ game. For this purpose, we apply a quadratic programming algorithm that allows us to calculate benchmarks for different parameter settings. (ii) We conduct a laboratory experiment and let participants decide in the firms' stead. Using the former calculated benchmark for the equilibrium price choices, we analyze the observed prices in the repeated game.

The experimental data support the intuition of mixed strategies. Among subjects' price choices, we find price decreases as well as price increases. Additionally, we observe high prices less frequently than low ones. Although we find disparities between the actual distribution of subjects' prices and the theoretical expectations, a statistical test reveals these differences not to be significant.
\end{abstract}

Keywords: Price-quantity competition, mixed strategy equilibrium, quadratic programming, experimental economics 


\section{INTRODUCTION}

Classical oligopoly analysis starts with quantity competition in Cournot (1838) and price competition in Bertrand (1883). In these papers, the basic mathematical concept for analyzing competition is established. The intersection of the so-called reaction functions yields a stable point in these models. A deviation from this equilibrium does not yield additional profit for either of the players. In the popular work of Nash (1951) this idea is further developed and the concept of Nash equilibrium is finally established. Besides pure strategy equilibria, i.e. equilibria with each player deciding on a unique strategy, Nash (1951) also derives mixed strategy equilibria with players choosing their strategies according to a probability distribution.

In economic research, we find several examples for mixed strategy equilibria in oligopoly games. Dixon (1984) presents a duopoly game that incorporates price choices for weakly convex cost curves and production levels calculated according to the sold units. In his game, the existence of a mixed strategy equilibrium in the symmetric case can be proved. Moreover, Maskin (1986) establishes the existence of a mixed strategy equilibrium even in case of asymmetric firms and more general cost structures in a game with capacity constraint price competition. Our model, however, focuses on the pure simultaneous price and quantity (PQ) choice in oligopolies. Levitan and Shubik (1978) analyze a similar game, but with a fixed unit cost for unsold quantity. They derive an analytical solution for the mixed strategy equilibrium. The main equilibrium properties for our game, however, are established by Gertner (1986).

In order to prove whether the mixed strategy prediction for our game is stable under laboratory conditions, we first transform the continuous strategy space into a discrete one. We then numerically calculate the probability distribution over prices that establishes a mixed strategy equilibrium for the discretized game. Using this as a benchmark, we set up a small experiment, where subjects were asked to choose prices and quantities. We compare the prices chosen during the experiment with the calculated numerical benchmarks and discuss similarities and differences.

This paper is organized as follows: The mathematical prerequisites for our analysis are reviewed in Section 2. We present a discrete version of the PQ-duopoly in Section 3. Our experimental analysis is described in Section 4 and Section 5 provides a concluding discussion of our findings.

\section{THE MIXED STRATEGY EQUILIBRIUM}

In a general setting, the game we are considering here includes $n$ firms denoted by $i=1, \ldots, n$. These firms decide on their prices $p_{i}$ and quantities $q_{i}$ simultaneously. The lowest-price firm $L$ can sell its full quantity up to the corresponding market demand $D\left(p_{L}\right)$. Under the assumption of efficient rationing, the remaining firms can satisfy the residual market demand in order of ascending prices. All firms have to overcome the symmetric production costs $C(q)$ depending on the number of articles produced. If two or more firms choose the same price, the demand is split equally between them depending on their respective production limits. For the duopoly situation, these informal rules define the payoff $\pi_{i}$ of player $i$ through

$$
\pi_{i}\left(p_{1}, q_{1}, p_{2}, q_{2}\right)=-C\left(q_{i}\right)+ \begin{cases}p_{i} \min \left[q_{i}, D\left(p_{i}\right)\right] & \text { if } p_{i}<p_{j} \\ p_{i} \min \left[q_{i}, D\left(p_{i}\right)-\min \left\{q_{j}, D\left(p_{j}\right)\right\}\right] & , \text { if } p_{i}>p_{j} \\ p_{i} \min \left[q_{i}, D\left(p_{i}\right)-\min \left\{q_{j}, \frac{D\left(p_{j}\right)}{2}\right\}\right] & , \text { if } p_{i}=p_{j}\end{cases}
$$

where $i, j=1,2 i \neq j$. For the purpose of our following experimental investigation, we consider a simplified version of this game with constant marginal production cost and a linear demand function. Hence,

$$
D(p)=a-p \quad \text { and } \quad C(q)=c q,
$$

where $a$ and $c$ are exogenously given constants. For this case of constant marginal production cost, Gertner (1986) shows that a symmetric mixed strategy equilibrium exists and that every PQ choice with a positive probability in this equilibrium satisfies $q_{i}=D\left(p_{i}\right)$. Thus, in equilibrium firms always choose a quantity equal to the market demand corresponding to their chosen price. Furthermore, Gertner (1986) 
proves that the probability distribution for the equilibrium prices is identical for both players and takes the form

$$
F(p)= \begin{cases}0 & , \text { for } p \in[0, c) \\ 1-c / p & , \text { for } p \in[c, a) \\ 1 & , \text { for } p \in[a, \infty)\end{cases}
$$

This probability distribution incorporates two types of outcomes: (i) with probability $1-c / a$, the player chooses to enter the competition and his price choice is distributed in a way that favors lower prices over higher ones. (ii) With probability $c / a$, he and chooses $p=a$ and therefore does not take place in the competition.

\section{A DISCRETE VERSION OF THE GAME}

To establish numerical benchmarks for games of the type presented in Section 2, we now consider a discrete game that only includes a finite number of possible prices from which each of the firms have to choose instead of a continuous space of prices. For the computation of mixed strategy equilibria in discrete games we follow the ideas of McCulloch (2011).

In the discrete game, each player can choose between a finite number of prices $p^{(k)}$ with $k=1, \ldots, N$. Following Gertner (1986), the production level is fixed at $q_{i}=D\left(p_{i}\right)$. A mixed strategy for this game is no longer described by a continuous probability distribution $F(p)$ but by the discrete probabilities of each of the choices. Let therefore $x_{i}^{(k)} \in[0,1]$ be the probability that firm $i=1,2$ chooses the price $p^{(k)}$. These probability values satisfy the condition

$$
\sum_{k=1}^{N} x_{i}^{(k)}=1, \quad i=1,2 .
$$

To simplify the notation, we define the strategy vector $\mathbf{x}_{\mathbf{i}}$ of firm $i$ as

$$
\mathbf{x}_{\mathbf{i}}=\left[x_{i}^{(1)}, \ldots, x_{i}^{(N)}\right]^{T} \in \mathbb{R}^{N}, \quad i=1,2 .
$$

We now define the payoff matrix $\mathbf{M}_{\mathbf{i}} \in \mathbb{R}^{N \times N}$ of player $i$ to be

$$
\mathbf{M}_{\mathbf{i}}=\left[\pi_{i}\left(p^{(k)}, D\left(p^{(k)}\right), p^{(l)}, D\left(p^{(l)}\right)\right)\right]_{k, l} \in \mathbb{R}^{N \times N},
$$

where $\pi_{i}$ is the payoff function given in (1). With these payoff matrices $\mathbf{M}_{i}$ and the strategy vectors $\mathbf{x}_{i}$, we can now define the optimization problem firm $i$ is facing as

$$
\max _{\mathbf{x}_{\mathbf{i}}} \mathbf{x}_{\mathbf{1}}{ }^{T} \mathbf{M}_{\mathbf{i}} \mathbf{x}_{\mathbf{2}} \quad \text { w.r.t. } \quad \mathbf{e}^{T} \mathbf{x}_{\mathbf{i}}=1 \quad \text { and } \quad \mathbf{x}_{\mathbf{i}} \geq 0
$$

where $\mathbf{e} \in \mathbb{R}^{N}$ is a vector of ones. In Mangasarian and Stone (1964), it is shown that the mixed-strategy equilibrium for this problem can be found by solving the optimization problem

$$
\begin{array}{r}
\max _{\mathbf{x}_{\mathbf{1}}, \mathbf{x}_{\mathbf{2}}, \alpha, \beta} \mathbf{x}_{\mathbf{1}}^{T}\left(\mathbf{M}_{\mathbf{1}}+\mathbf{M}_{\mathbf{2}}\right) \mathbf{x}_{\mathbf{2}}{ }^{T}-\alpha-\beta, \quad \text { w.r.t. } \\
\mathbf{M}_{\mathbf{1}} \mathbf{x}_{\mathbf{2}} \leq \alpha \mathbf{e} \text { and } \quad \mathbf{M}_{\mathbf{2}}{ }^{T} \mathbf{x}_{\mathbf{1}} \leq \beta \mathbf{e} \quad \text { and } \\
\mathbf{x}_{\mathbf{1}}{ }^{T} \mathbf{e}=\mathbf{x}_{\mathbf{2}}{ }^{T} \mathbf{e}=1 \quad \text { and } \quad \mathbf{x}_{\mathbf{1}}, \mathbf{x}_{\mathbf{2}} \geq 0
\end{array}
$$

This problem can be solved using quadratic programming as is explained in Mangasarian and Stone (1964).

We implemented a numerical solution for this problem in order to be able to analyze different discretized versions of our game and compare them to the experimental outcome. Our implementation takes as inputs the system parameters $a, c$ as well as the discretization parameter $N$. The prices $p^{(k)}$ are generated by dividing the interval $[c, a]$ in $N-1$ equally distributed subintervals. Figure 1 shows a comparison between 


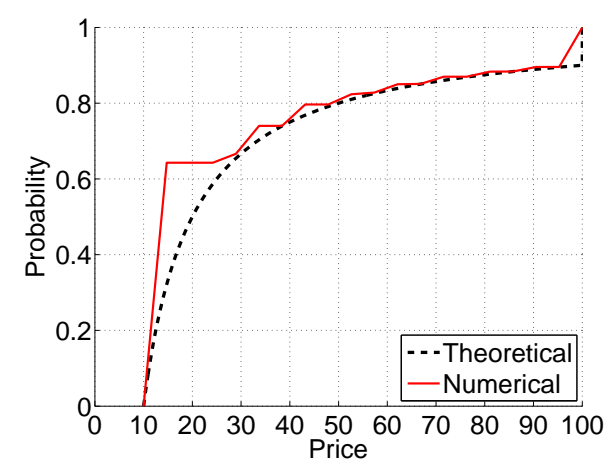

(a) $N=20$

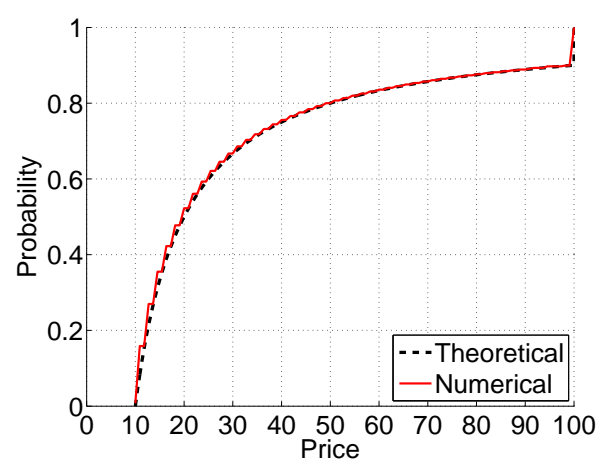

(b) $N=100$

Figure 1. Mixed strategy equilibria - discrete game (solid red line) vs. continuous game (dashed black line)

the mixed strategy equilibria of the continuous and the discrete game. Keeping the game parameters fixed as $a=100$ and $c=10$, we varied the discretization parameter, choosing $N=20$ and $N=100$. The dashed black line hereby displays the distribution given in (2). We can see that for small $N$ the equilibria differ visibly while for increasing $N$ the difference decreases rapidly. We therefore conclude that the mixed strategy equilibrium in the discrete version of our game is comparable to that in the continuous game.

\section{THE EXPERIMENT}

For our experimental analysis, we invited 22 students to the Magdeburg laboratory of experimental economics (MaXLab). The MaXLab is part of the faculty of Economics and Management at the University of Magdeburg. We used the online recruitment system ORSEE of Greiner (2004) and guaranteed that the invited students had at least some economic background. The majority of them were economics students. Out of the 22 students initially invited, two were reserve, to ensure enough subjects would be present. As all invited subjects came to the experiment, we randomly chose 20 to participate in the experiment. The remaining two students were compensated for their arrival. With these 20 subjects we were therefore able to collect ten independent pairwise observations.

The experiment was run in two sessions in June 2011. After validating their identity, we advised each subject to take a seat in front of a computer. Without subjects' knowledge, computers were randomly linked to form the pairs for our game. In the following, communication between the subjects was strictly prohibited to avoid collusion effects. We gave the instructions to the subjects and read them aloud and answered questions individually to make sure that every subject was completely aware of the experimental design. From the start of the experiment, each session lasted about 45 minutes.

\subsection{EXPERIMENTAL DESIGN}

Our game is well aligned with the model presented in Section 2. It includes a duopoly setting with two firms deciding on their market price $p$ as well as on their production quantity $q$. At the beginning of the experiment, each subject was randomly assigned one of two firms. We assigned labels "Firm $A$ " and "Firm $B$ " to the firms. However, the two firms are completely identical.

We introduced a simple pre-game, to control for location specific bias, i.e. to be sure that our experimental results are comparable and are not affected by the laboratory environment itself in a particular way. We therefore situated each subject individually into a monopoly setting with the given demand function $D(p)=100-p$. Subjects had to choose the market price in the range $p \in[0,100]$. For technical reasons, we limited the increment to 0.001 . Additionally, subjects had to choose a production quantity in the range $q \in[0,100]$. We used the same increment of 0.001 . Subjects were not allowed to produce more than the demand corresponding to the chosen price. Total production cost equaled $10 q$. 
We let the subjects play five rounds of this simple monopoly game. At the beginning of each round, we endowed subjects with a calculator program. Subjects could enter various combinations of market prices and production quantities and calculate corresponding payoffs. At the end of each round, subjects' payoffs were calculated as $\pi=(p-10) q$. A summary of the price, the quantity and the corresponding payoff in this round and the cumulated payoff was presented. Subjects' total payoff for the pre-game was calculated as the sum of the payoff from all five rounds.

For the duopoly game, we situated both firms in one common market. Again, subjects had to choose their market price and their production quantity from the range $[0,100]$ with 0.001 increments. The production quantity was again limited by the corresponding market demand and production cost were still 10q. The demand for each firm in this duopoly game depended on the subjects' decisions on their prices as well as on their quantities. The number of sold units and the payoffs for both firms were calculated according to the model presented in Section 2.

The duopoly game was played for 20 rounds. At the beginning of each round, subjects were given a calculator program to calculate payoffs for various combinations of market prices and production quantities of both firms. We therefore guaranteed that subjects could get used to the calculation of residual demand and payoffs. After their simultaneous decisions on prices and quantities, subjects' payoffs were calculated according to (1). At the end of each round of the duopoly game, we informed the subjects about the price and quantity the other firm had chosen and summarized the round results. Subjects' total payoffs from the duopoly game were finally calculated as the sum of the payoff from each of the 20 rounds.

At the end of the experiment, instructions were collected and the subjects were anonymously paid according to their cumulated payoffs from both, the monopoly game and the duopoly game. We fixed the exchange rate of the game payoffs at $1,500=1 €$. Subjects mean earnings were $\mu=10.31 €$ (at this time $\approx 14.74 \$$ ). The highest payoff in our game was $20.40 €$, the lowest was $0 €$ due to bankruptcy. Payoffs' standard deviation can be calculated as $\sigma=4.91 €$.

\subsection{RESULTS}

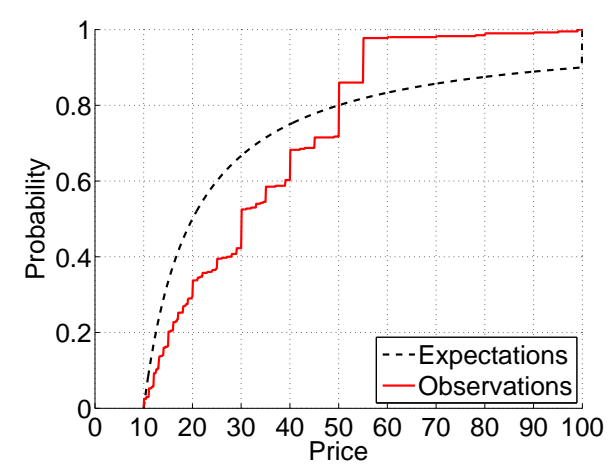

Figure 2. Distribution of prices - subjects' decisions (solid red line) vs. mixed strategy equilibrium (dashed black line)

Figure 2 displays the distribution of the subjects' price choices. We can see that the distribution looks somewhat similar to the numerical benchmarks, but some differences can be spotted as well. As shown in table 1, we observe that lower prices are chosen more frequently than higher prices, which is in line with lower prices having a higher probability in the mixed strategy equilibrium. A second similarity between the observed and the predicted distribution concerns the lowest price choice made during the experiments. With the exception of one observation of $p_{\min }=9.5$ the lower bound of chosen prices was the marginal cost level $c=10$. Additionally, Table 2 shows that prices were chosen non-monotonously, as we observe price increases and decreases regularly. 
Table 1. Subjects' price choices

\begin{tabular}{lcccc}
\hline & $p<10$ & $10 \leq p<25$ & $25 \leq p<40$ & $40 \leq p<55$ \\
\hline observed frequency & 1 & 147 & 93 & 103 \\
expected frequency & 0 & 240 & 60 & 27 \\
\hline \hline & $55 \leq p<70$ & $70 \leq p<85$ & $85 \leq p<100$ & $p=100$ \\
\hline observed frequency & 48 & 5 & 3 & 0 \\
expected frequency & 16 & 10 & 7 & 40
\end{tabular}

Table 2. Subjects' price changes

\begin{tabular}{cccc}
\hline & price decreases & price increases & no price change \\
\hline observed frequency & 172 & 94 & 114 \\
absolute average change & -9.79 & 14.75 & 0 \\
relative average change & $-26 \%$ & $89 \%$ & $0 \%$ \\
\hline
\end{tabular}

In contrast to the theoretical predictions, we do not observe prices equal to 100 . The highest price that was chosen in our experiment was $p_{\max }=99$. We conclude that subjects never decided to refrain from competition because they expected average round payoffs to be positive. Overall, when we compare the frequency of subjects price choices with its expected frequencies in intervals as displayed in Table 1, we find no strong significant difference (Kolmogorov-Smirnov One-Sample Test, $p=0.0815$ ). This indicates that observed frequencies could reasonably present a random deviation from the expected distribution.

\section{CONCLUSIONS}

This paper establishes, on the one hand, a mathematical guide for creating numerical benchmarks for duopoly games with mixed strategy equilibria. We are able to show that for a simple game with simultaneous price and quantity choice, the mixed strategy equilibrium of a discretized game is well aligned with the theoretical predictions for the corresponding continuous game. We implemented a quadratic programming algorithm for the discretized game in order to calculate benchmarks for various parameter values.

One the other hand, we conducted an experimental investigation of the same simple game. We found similarities between our experimental results and the theoretical prediction of the mixed strategy equilibrium. We observed a non-monotonous price choice from an expected range of prices. The difference between observed and expected prices was found not to be significant. These results indicate that subjects in our experiments acted according to a mixed strategy that incorporates similar features as the predicted equilibrium. Therefore, a similar method of analyzing mixed strategy equilibria in numerical and experimental settings can be applied to a wide range of oligopoly games, e.g. capacity constrained duopolies or duopolies with inventories.

Finally, according to Erev and Roth (1998), we would like to remark that theory has not always the greatest predictive power in games with unique mixed strategy equilibria. Erev and Roth (1998) argue that the data of several repeated games they analyzed could be better understood when learning models are considered. We are therefore encouraged to look at our data considering learning effects and behavioral patterns in a further work.

\section{ACKNOWLEDgment}

We would like to thank Abdolkarim Sadrieh for helpful suggestions and comments. The first author would like to thank the financial support from the Chair in E-Business, University of Magdeburg. The second 
author would like to thank the financial support from the European Research Council under the European Community's Seventh Framework Programme (FP7/2007-2013) / ERC grant agreement No. 239870. His work is also partly supported by Award No KUK-C1-013-04, made by King Abdullah University of Science and Technology (KAUST).

\section{REFERENCES}

Bertrand, J. L. F. (1883). Review of Théorie Mathématique de la Richesse Sociale and Recherches sur les Principes Mathématique de la Théorie des Richesse. Journal des Savants 67, 499-508.

Cournot, A. A. (1838). Researches into the Mathematical Principles of the Theory of Wealth economics. Paris: Librairie des sciences politiques et sociales M. Riveáre \& cie. / translated by N. Bacon, New York: Macmillan Company, 1927.

Dixon, H. (1984). The existence of mixed-strategy equilibria in a price-setting oligopoly with convex costs. Economics Letters 16(3-4), $205-212$.

Erev, I. and A. E. Roth (1998). Predicting how people play games: Reinforcement learning in experimental games with unique, mixed strategy equilibria. The American Economic Review 88(4), 848-881.

Gertner, R. (1986). Essays in Theoretical Industrial Organization. Ph. D. thesis, Princeton University.

Greiner, B. (2004). The online recruitment system orsee 2.0 - a guide for the organization of experiments in economics. Working Paper Series in Economics 10, University of Cologne, Department of Economics.

Levitan, R. E. and M. Shubik (1978). Duopoly with price and quantity as strategic variables. International Journal of Game Theory 7(1), 1-11.

Mangasarian, O. L. and H. Stone (1964). Two-person nonzero-sum games and quadratic programming. Journal of Mathematical Analysis and Applications 9, 348-355.

Maskin, E. (1986). The existence of equilibrium with price-setting firms. The American Economic Review 76(2), 382-386.

McCulloch, H. (2011). Pq-nash duopoly: A computational characterization. Submitted.

Nash, J. (1951). Non-cooperative games. The Annals of Mathematics 54(2), 286-295. 